\title{
Comparison of the use of ventricular access devices and ventriculosubgaleal shunts in posthaemorrhagic hydrocephalus: systematic review and meta-analysis
}

\author{
Daniel M. Fountain ${ }^{1}$ - Aswin Chari ${ }^{2,3}$ • Dominic Allen ${ }^{4}$ - Greg James ${ }^{5,6}$
}

Received: 11 September 2015 / Accepted: 29 October 2015 /Published online: 11 November 2015

(C) The Author(s) 2015. This article is published with open access at Springerlink.com

\begin{abstract}
Introduction Ventricular access devices (VAD) and ventriculosubgaleal shunts (VSGS) are currently both used as temporising devices to affect CSF drainage in neonatal posthaemorrhagic hydrocephalus ( $\mathrm{PHH}$ ), without clear evidence of superiority of either procedure. In this systematic review and meta-analysis, we compared the VSGS and VAD regarding complication rates, ventriculoperitoneal shunt conversion and infection rates, and mortality and long-term disability.

Methods The review was registered with the PROSPERO international prospective register of systematic reviews (registration number CRD42015019750) and was conducted in accordance with PRISMA guidelines.

Results and conclusions The literature search of five databases identified 338 publications, of which 5 met the inclusion criteria.
\end{abstract}

Electronic supplementary material The online version of this article (doi:10.1007/s00381-015-2951-8) contains supplementary material, which is available to authorized users.

Aswin Chari

aswinchari@gmail.com

1 School of Clinical Medicine, University of Cambridge, Cambridge, UK

2 Victor Horsley Department of Neurosurgery, National Hospital for Neurology and Neurosurgery, London, UK

3 Division of Brain Sciences, Faculty of Medicine, Imperial College London, 5th Floor, Burlington Danes Building, Du Cane Road, London W12 0NN, UK

4 School of Medicine, Imperial College London, London, UK

5 Department of Neurosurgery, Great Ormond Street Hospital, London, UK

6 Developmental Neurosciences Programme, Institute of Child Health, University College London, London, UK
All were retrospective cohort studies (evidence class $3 b$ and 4). A significantly lower proportion of patients with a VSGS required CSF tapping compared to patients with a VAD (log OR $-4.43,95 \% \mathrm{CI}-6.14$ to -2.72 ). No other significant differences between the VAD and VSGS were identified in their rates of infection ( $\log$ OR $0.03,95 \% \mathrm{CI}-0.77$ to 0.84 ), obstruction ( $\log$ OR $1.25,95 \% \mathrm{CI}-0.21$ to 2.71 ), ventriculoperitoneal shunt dependence $(\log \mathrm{OR}-0.06,95 \% \mathrm{CI}-0.93$ to 0.82$)$, subsequent shunt infection ( $\log$ OR $0.23,95 \% \mathrm{CI}-0.61$ to 1.06 ), mortality ( $\log$ OR $0.37,95 \% \mathrm{CI}-0.95$ to 1.70 ) or long-term disability $(p=0.9)$. In all studies, there was a lack of standardised criteria, variations between surgeons in heterogeneous cohorts of limited sample size and a lack of neurodevelopmental follow-up. This affirms the importance of an ongoing multicentre, prospective pilot study comparing these two temporising procedures to enable a more robust comparison.

Keywords Intraventricular haemorrhage · Posthaemorrhagic hydrocephalus · Ventricular access device .

Ventriculosubgaleal shunt

\section{Introduction}

Preterm infants, particularly those classed as "extremely low birth weight" $(<1000 \mathrm{~kg})$, are at risk of bleeding from the germinal matrix of the developing brain, resulting in intraventricular haemorrhage (IVH). Depending on the grade of IVH, $25-80 \%$ of affected infants develop radiological posthaemorrhagic ventricular dilatation (PHVD) and clinical evidence of posthaemorrhagic hydrocephalus (PHH) [1]. Preterm IVH is an important clinical problem in these children: PHH has been associated with significantly impaired long-term neurodevelopment [2]. PHH has been shown to result in a three-fold increase in cognitive and 
psychomotor delay, with nearly a third of patients suffering from epilepsy [3]. Furthermore, IVH is an independent risk factor for cerebral palsy $[4,5]$.

The definitive treatment for PHH is CSF diversion achieved by insertion of a ventriculoperitoneal shunt (VPS). Insertion of VPS is discouraged in infants weighing less than $2 \mathrm{~kg}$ due to immunological immaturity, technical factors and the risk of abdominal sepsis [6, 7]. They may also often have significant co-morbidities, including sepsis, respiratory impairment and abdominal complications such as necrotising enterocolitis [4]. In addition, a proportion of infants may not require permanent CSF drainage after clearance of the intraventricular blood $[8,9]$. Therefore, in the interim, progressive symptomatic ventricular dilatation is often treated with temporary CSF diversion, with measures including lumbar punctures, ventricular tapping, external ventricular drains (EVDs) and the so-called temporising devices (TDs-see below). In previous systematic analyses, early repeated CSF tapping using lumbar punctures and ventricular tapping could not be recommended; lumbar punctures did not change outcomes in comparison to observation $[10,11]$. The two most wellestablished TDs are a ventricular access device (VAD) and a ventriculosubgaleal shunt (VSGS). A VAD involves insertion of a subcutaneous reservoir connected to a ventricular catheter for percutaneous CSF tapping [12]; typically, these are aspirated percutaneously at regular intervals (e.g. every $12-48 \mathrm{~h}$ ) in order to maintain head circumference [13]. A VSGS is a CSF shunt with the ventricular catheter draining directly into a subgaleal scalp pocket, created during the surgical procedure $[4,14]$. There is evidence that VADs reduce morbidity and mortality compared with EVDs [11].

There are theoretical advantages in using a VSGS instead of a VAD; the VSGS permits resorption through a subgaleal scalp pocket, reducing the need for intermittent tapping required with a VAD $[12,14]$. The VSGS also establishes a permanent decompression without causing electrolyte and nutritional losses [15]. However, potential complications of the use of a VSGS include scarring of the subgaleal pocket $[14,16]$, and/or CSF leakage [16-18], and some series report significant infection and failure rates $[17,19]$.

Currently, there is no strong evidence favouring the use of one particular TD over another, and the choice of which to use in a particular case is often down to the experience and preference of the treating neurosurgeon. Differences in outcomes between VADs and VSGSs as for the management of PHH remain poorly understood [11]. Therefore, this systematic review sought to compare VAD and VSGS in key outcomes such as complication rate (including infection and failure), permanent VPS requirement rate, long-term disability and mortality in neonates with PHH.

\section{Methods}

This systematic review was conducted according to the PRISMA (Preferred Reporting Items for Systematic Reviews and Meta-Analyses) guidelines and has been registered with the PROSPERO international prospective register of systematic reviews (registration number CRD42015019750). A systematic search of keywords in Table 1 was performed independently by two authors (DMF and DA) of MEDLINE Complete via EBSCOhost, EMBASE 1974 to 2015 via Ovid, the Cochrane Central Register of Controlled Trials (CENTRAL) via the Cochrane Library and ClinicalTrials.gov databases on the 22nd April 2015. A record of our MEDLINE Complete search is provided in the Electronic Supplementary Material (Table S1).

Initially, titles and abstracts were screened for relevant papers. The full texts were then attained and reviewed. Both processes were undertaken by two authors independently (DMF and DA). Decisions were blinded and, where disagreements occurred, both authors discussed the disparities and resolved them throughout the selection process. Data extraction was also performed by two authors (DMF and DA) to ensure reliability. Where inconsistent reporting formats of data were published, authors were contacted directly to enable collection of comparable data using a standardised data collection template (Electronic Supplementary Material Table S2). The inclusion criteria were as follows:

1. Study design: Peer-reviewed published original research. Abstracts, commentaries, reviews and research without peer review were excluded.

2. Population: At least 10 patients with posthaemorrhagic hydrocephalus of prematurity (PHH).

3. Intervention: Use of ventricular access device (VAD) and ventriculosubgaleal shunt (VSGS) in a comparative study.

4. Outcome: Results including at least one of the following:

Table 1 Search terms used in the literature review

\begin{tabular}{lll}
\hline Population & Problem & Intervention \\
\hline Infant & Hemorrhage & Ommaya Reservoir* \\
Infant, Newborn & Hydrocephalus & VAD \\
Infant* & Hemorrhag* & Ventricular Access Device* \\
Neonat* & Haemorrhag* & Ventricular Reservoir* \\
& Intraventricular & Subcutaneous Reservoir* \\
& Intra Ventricular & VSGS \\
& Posthemorrhagic & Subgaleal Shunt* \\
& Posthaemorrhagic & Ventriculosubgaleal Shunt* \\
& Post Hemorrhagic & \\
& Post Haemorrhagic & \\
\hline
\end{tabular}

Medical Subject Headings (MeSH) are shaded in grey. Booleans "OR" and "AND" were utilised to combine row and column terms, respectively 

a. Rate of TD infection and obstruction
b. VPS conversion rate
c. Subsequent VPS infection
d. Mortality.

Evidence classification for accepted studies was performed based on the Oxford Centre for Evidence-based MedicineLevels of Evidence [20]. All studies were appraised for their quality of reporting using the STROBE statement alongside separate evaluation of methods and validity of conclusions [21]. Where reported, data on each major outcome was converted to a log odds ratio (OR) with $95 \%$ confidence interval (CI) and combined across studies in a meta-analysis. Reporting of meta-analysis was undertaken in accordance with the proposed checklist published by the Meta-analysis Of Observational Studies in Epidemiology (MOOSE) group [22]. Pooling of OR estimates was performed using an inverse variance weight random-effects DerSimonian-Laird meta-analysis, with Cochran's $Q$ test for heterogeneity [23]. If the $Q$ test value was less than $(k-1)$, where $k=$ number of studies, fixed-effects method was also reviewed for consistency of results as a sensitivity analysis. All statistical analysis was performed using the metafor package in $\mathrm{R}$, version 3.0.2 [24, 25].

A flow diagram for the results of the systematic search process is provided in the Electronic Supplementary Material (Figure S1). Forward and backward searching of accepted papers was also performed to identify additional studies not captured in the systematic search results. The search was repeated on 19th August 2015. No additional studies were identified.

\section{Results}

Five original research studies were identified, all retrospective cohort studies (Table 2). Three studies [8, 26, 27] were classified as class $3 \mathrm{~b}$ due to tested homogeneity of birth weight and gestational age between cohorts. Wang et al. tested the cohorts and found them to be significantly heterogeneous, whereas Wellons et al. conducted no testing for homogeneity of cohorts [28, 29]. Both studies were thus assigned as evidence class 4. Meta-analysis of the published data where pooling was possible is presented in Fig.1. Across all outcome measures, Cochran's Q test for heterogeneity was nonsignificant $(p>0.05)$. Fixed effect sensitivity analysis provided consistent results where performed.

\section{Temporising device CSF taps, infection and obstruction}

Two studies evaluated CSF tapping. Wang et al. identified a significantly higher number of CSF taps in patients with VADs than VSGSs (VAD $10 \pm 8.7$ taps vs. VSGS $1.6 \pm 1.7$ taps, $p<0.001$ ) [28]. This was the case despite a significantly longer time from TD to VPS (VAD $48.8 \pm 26.4$ days vs. VSGS $80.8 \pm 67.5$ days, $p=0.012$ ). Furthermore, Lam and Heilman reported that $16 / 16$ patients with VADs required daily CSF tapping, compared to only 4/16 patients with VSGSs $(p=0.000016)$ [8]. Meta-analysis showed a significantly lower proportion of patients with a VSGS requiring CSF tapping compared to patients with a VAD (Fig. 1a, log OR -4.43, $95 \%$ CI -6.14 to -2.72 ). However, this did not translate to significantly higher rates of infection or obstruction with VADs compared to VSGSs in either study. Infection rates reported in four studies ranged from 0 to $12.5 \%$ for VADs and 6.3 to $13.9 \%$ for VSGSs [8, 27-29]. Meta-analysis showed no significant difference between VAD and VSGS rates of infection and obstruction (Fig. 1b, c; infection log OR $0.03,95 \% \mathrm{CI}-0.77$ to 0.84 ; obstruction $\log$ OR 1.25 , $95 \% \mathrm{CI}-0.21$ to 2.71 ). One study investigated the need for revision, finding no significant difference between revision rates in patients with a VAD and VSGS (VAD $3.1 \%$ vs. VSGS $10 \%, p=0.16$ ) [27].

\section{Ventriculoperitoneal shunt dependence}

The proportion of patients with a VAD and VSGS converted to VPS varied greatly between studies (58.5-93.8 \% for VADs and $60-86 \%$ for VSGSs). Whilst the Wellons et al. study found borderline significance in the number of patients with a VSGS requiring a VPS $(69.3 \%$ for VADs vs. $86.1 \%$ for VSGSs, $p=0.05$ ) [29], meta-analysis of the studies found no significant difference (Fig. 1d, log OR $-0.06,95 \%$ CI -0.93 to 0.82$)$. Subsequent VPS infection rates were comparable between patients with VADs (4.6-12\%) and VSGSs (3.3$16 \%)$, with no significant difference following metaanalysis (Fig. 1e, log OR 0.23, $95 \%-0.61$ to 1.06 ).

\section{Mortality}

Despite representing a core outcome in the management of posthaemorrhagic hydrocephalus and the preterm infant, only three studies reported mortality. In the studies, all mortalities were unrelated to the posthaemorrhagic hydrocephalus or complications relating to its management $[8,27,28]$. No significant difference in mortality was found between patients with VADs and VSGSs in the reporting studies and in meta-analysis of the data (Fig. 1f, $\log$ OR $0.37,95 \% \mathrm{CI}-0.95$ to 1.70 ).

\section{Long-term neurodevelopment}

Only one study reported neurodevelopmental outcome. Srinivasakumar et al. utilised the Bayley Scales of Infant and Toddler development, with results at 18 and 24 months for $36 \%$ of surviving infants. Comparative testing between patients with a VAD and VSGS was not significant $(p=0.9)$ [26]. 


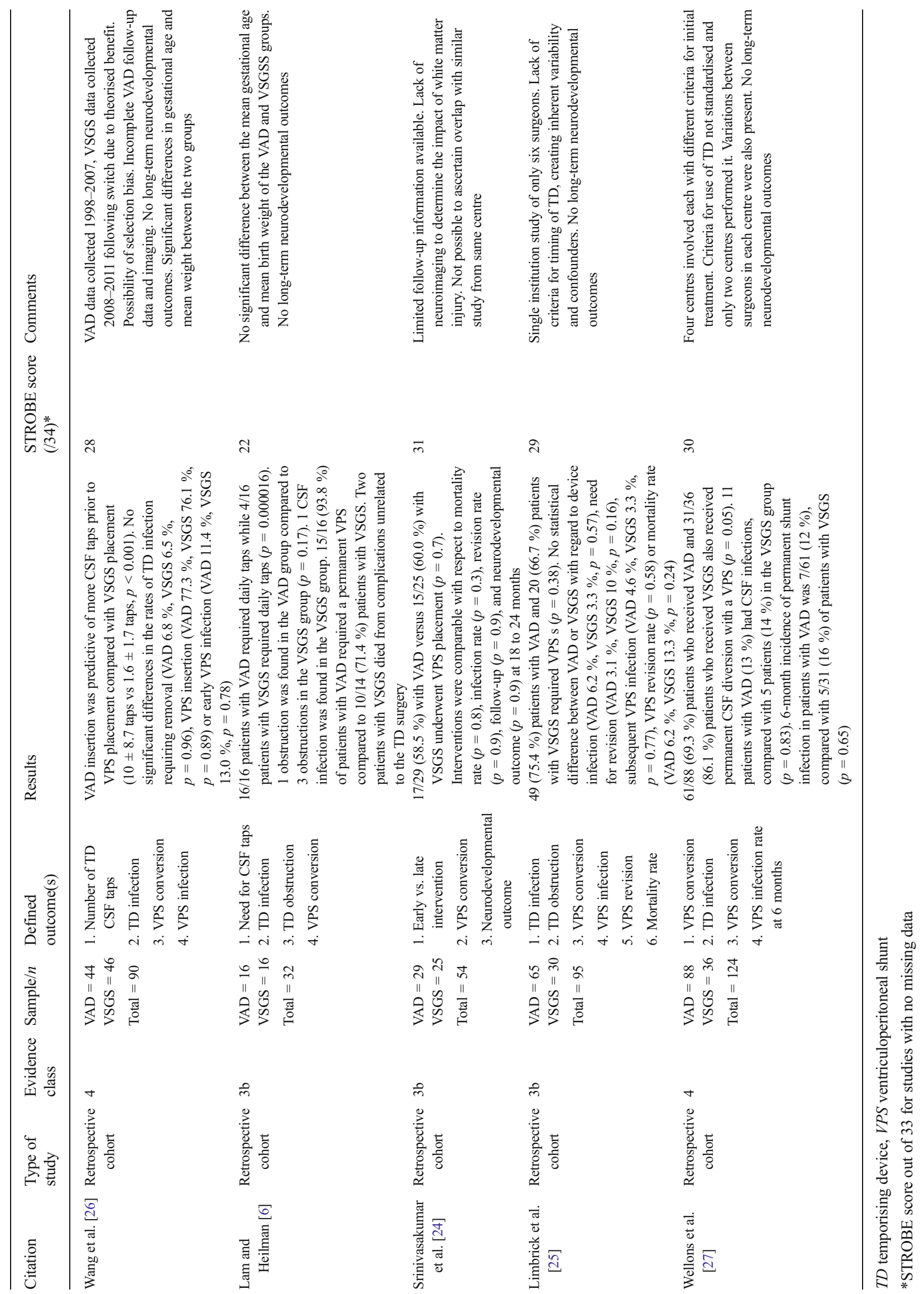




\section{Criteria}

Various criteria were reviewed relating to the decision to place a TD, the TD type, at which point(s) CSF tapping was indicated, when VPS conversion was indicated and patient inclusion in the studies (Table 3). TD placement criteria varied between studies, but common indications included clinical instability of the infant, identified PHVD or external signs of raised intracranial pressure. Two studies gave no detail relating to indications for TD placement. The decision on the type of TD was either due to surgeon training or preference, or the preference of the centre. Two studies evaluated centres that had transitioned all treatment from VAD to VSGS on the grounds of theorised benefits of the VSGS $[8,28]$.

Indications for CSF tapping included instability of vital signs, rapid increase in head circumference, signs of hydrocephalus on cranial ultrasonography and failure of adequate absorption of the subgaleal pocket in patients with VSGSs. Whilst one study reported routine tapping until signs of hydrocephalus resolved [8], two studies indicated CSF tapping as required $[27,28]$. The two remaining studies provided no clear details of CSF tapping protocols [26, 29]. For treatment of CSF infection, one study reported removing the device based on surgeon preference [28]. Specific VPS conversion criteria varied greatly between studies but included elements related to the weight and estimated gestational age (EGA) of the infant, vital sign deterioration, persisting PHVD and need for CSF tapping. Furthermore, criteria for patient inclusion in the study varied. Three studies specified patients with a diagnosed grade III or IV IVH [26, 27, 29]. Two studies specified a weight of less than $1500 \mathrm{~g}[27,29]$. Exclusion criteria included patients with congenital central nervous system malformations and stroke, and those who received care outside of the facility studied $[26,29]$. Where specified, EGA for the patients included ranged from 34 to 40 weeks [26-28].

\section{Discussion}

Identifying the optimal temporising device for PHH is important in limiting potentially devastating consequences from this complication of IVH. The decision to use either a VAD or VSGS remains poorly understood [11], and this is the first review to compare these temporising devices in a systematic way across multiple outcomes and meta-analyse the results where possible. Whilst criteria for diagnosing IVH were somewhat comparable in the characteristics reviewed, specific measures or thresholds for subsequent PPHVD were not reported in any included study. This finding corroborates a survey of neonatologists that demonstrated substantial heterogeneity of diagnosis and management of this condition across Europe [30].
For those studies that reported TD infection rates, two of the five studies included criteria for infection (CSF culture positivity) $[27,28]$. Although CSF tapping has been associated with an increased risk of infection [10], the results here demonstrate that, despite a significantly higher rate of tapping in patients with a VAD, there is no evidence to suggest a higher rate of infection of the VAD relative to the VSGS. Despite the theoretical risks, it is hypothesised that the use of rigorous protocols for CSF tapping minimises the infection rate for VADs [31]. Methods of CSF tapping used to minimise infection were not described, but the range of infection rates reported suggests potential differences between centres in absolute infection rates.

The significantly reduced rate of tapping may be an attractive feature of VSGS for neurosurgical units in certain localities. Whilst in North America these infants are likely to stay at the neurosurgical centre for the majority of their neonatal care, where tapping of the VAD can be supervised and performed by neurosurgical professionals, many European centres will discharge these children soon after surgery to their local neonatal unit for ongoing care. The lack of "control" over frequency and technique of tapping in peripheral hospitals may push these units towards the use of VSGS. Standard protocols for indications for CSF tapping were not consistently used across studies, including the clinical indications, timing of tapping and volume of CSF extracted. Furthermore, standard protocols for the treatment of CSF infection or TD obstruction were not described in the studies included in this review.

VPS conversion and subsequent infection was not significantly different between patients managed with a VAD or VSGS, but this finding is confounded by the heterogeneity in timing and criteria used to decide when to convert to a permanent VPS; the time interval from TD to VPS was analysed in only one study [28]. Similar to the TD infection rate, the VPS infection rate varied greatly between studies. Protocols for management of VPS infections were not described in the studies included in this review.

With regard to mortality and neurodevelopmental status, there is potential selection bias as Wellons et al. excluded patients who died and Limbrick et al. reported substantial comorbidities that contributed to the mortality rate [27, 29]. Furthermore, all of the aforementioned outcome measures are potential confounders, in particular TD and VPS infection. The length of follow-up in studies also varied greatly, with no standard criteria for the determination of a mortality rate reported. A multivariable model approach would better elucidate the factors contributing to long-term neurodevelopmental outcome.

The results affirm the importance of an ongoing multicentre, prospective pilot study comparing these two temporising procedures to enable a more robust comparison, with standardisation of protocols across diagnosis, TD insertion, VPS conversion and measurement of long-term outcome [32]. 
Fig. 1 Meta-analysis of VSGS relative to VAD. Statistics presented are Cochran's $Q$ with $p$ value test for heterogeneity and $\log$ odds ratio (OR) with $95 \%$ confidence intervals (CI). An asterisk represents the results for VPS conversion rate from Srinivasakumar et al. [26] excluded due to potential overlap with Limbrick et al. [27]

\section{VSGS relative to VAD - Meta-Analysis}

a. TD CSF Taps Required $-n=122, Q=0.0047, p=0.9452$

\begin{tabular}{llllll}
\hline Wang et al. 2014 \\
Lam and Heilman 2009
\end{tabular}

b. TD Infection - $n=341, Q=0.8423, p=0.8393$

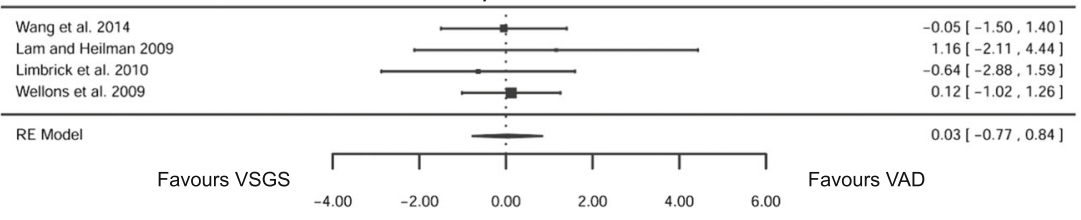

C. TD Obstruction $-n=127, Q=0.0001, p=0.9943$

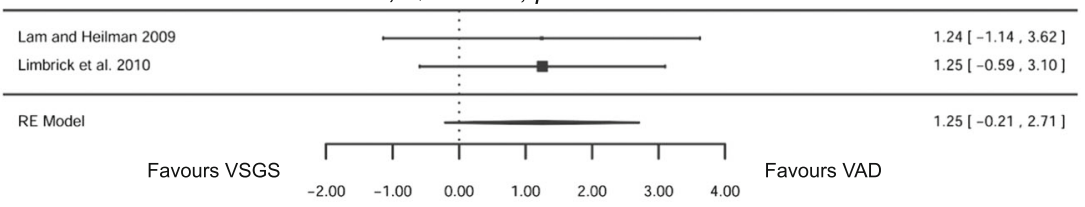

d. VPS Required - $n=339^{*}, Q=6.6282, p=0.0847$

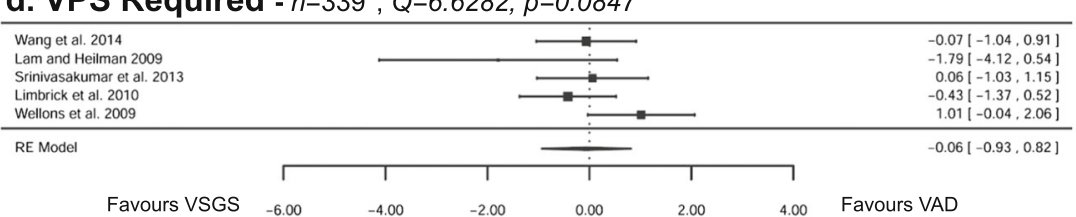

e. VPS Infection $-n=230, Q=0.2131, p=0.8989$

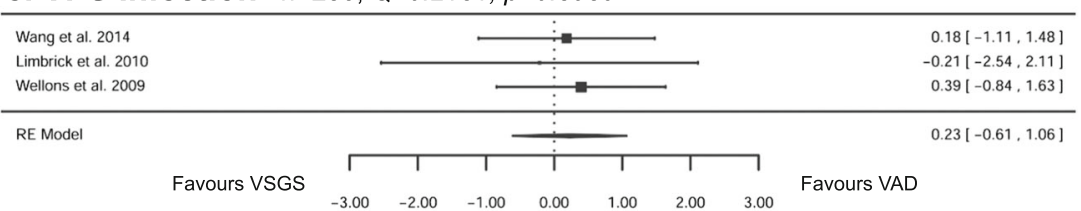

f. Mortality $-n=217, Q=2.8424, p=0.2414$

\begin{tabular}{|c|c|c|c|c|c|c|c|c|}
\hline \multirow{3}{*}{$\begin{array}{l}\text { Wang et al. } 2014 \\
\text { Lam and Heilman } 2009 \\
\text { Limbrick et al. } 2010\end{array}$} & & 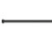 & $\vdots$ & & & & & \multirow{3}{*}{$\begin{array}{r}-0.79[-2.54,0.96] \\
1.74[-1.38,4.86] \\
0.85[-0.61,2.31]\end{array}$} \\
\hline & & & $\vdots$ & & & & & \\
\hline & & & : & $\longrightarrow$ & & & & \\
\hline \multirow{3}{*}{ Favours VSGS } & & & - & & & & \multirow{3}{*}{ Favours VAD } & \multirow[t]{3}{*}{$0.37[-0.95,1.70]$} \\
\hline & Г & $T$ & $T$ & $T$ & $T$ & 7 & & \\
\hline & -4.00 & -2.00 & 0.00 & 2.00 & 4.00 & 6.00 & & \\
\hline
\end{tabular}

\section{Limitations}

The absences of a rationale for the sample size, a participant flow diagram or the use of sensitivity analysis were key omissions that reduced the STROBE scores for the included studies. Major specific limitations identified in the studies include heterogeneity of patient cohorts [28], lack of neurodevelopmental follow-up [8, 27-29], absence of standardised criteria for the management of this condition and variations between surgeons creating inherent variability and confounders [27, 29]. Limited sample sizes reduced statistical power in all studies. The meta-analysis presented gives estimates across core outcomes to provide results from a larger sample. However, whilst heterogeneity was assessed statistically in the meta-analysis, it was nonetheless performed on a series of retrospective studies with substantial heterogeneity in the criteria for management and reporting of outcomes in patients with posthaemorrhagic hydrocephalus.

\section{Conclusion}

This study reports, in an objective and systematic fashion, the current state of the literature regarding which TD (VAD or 


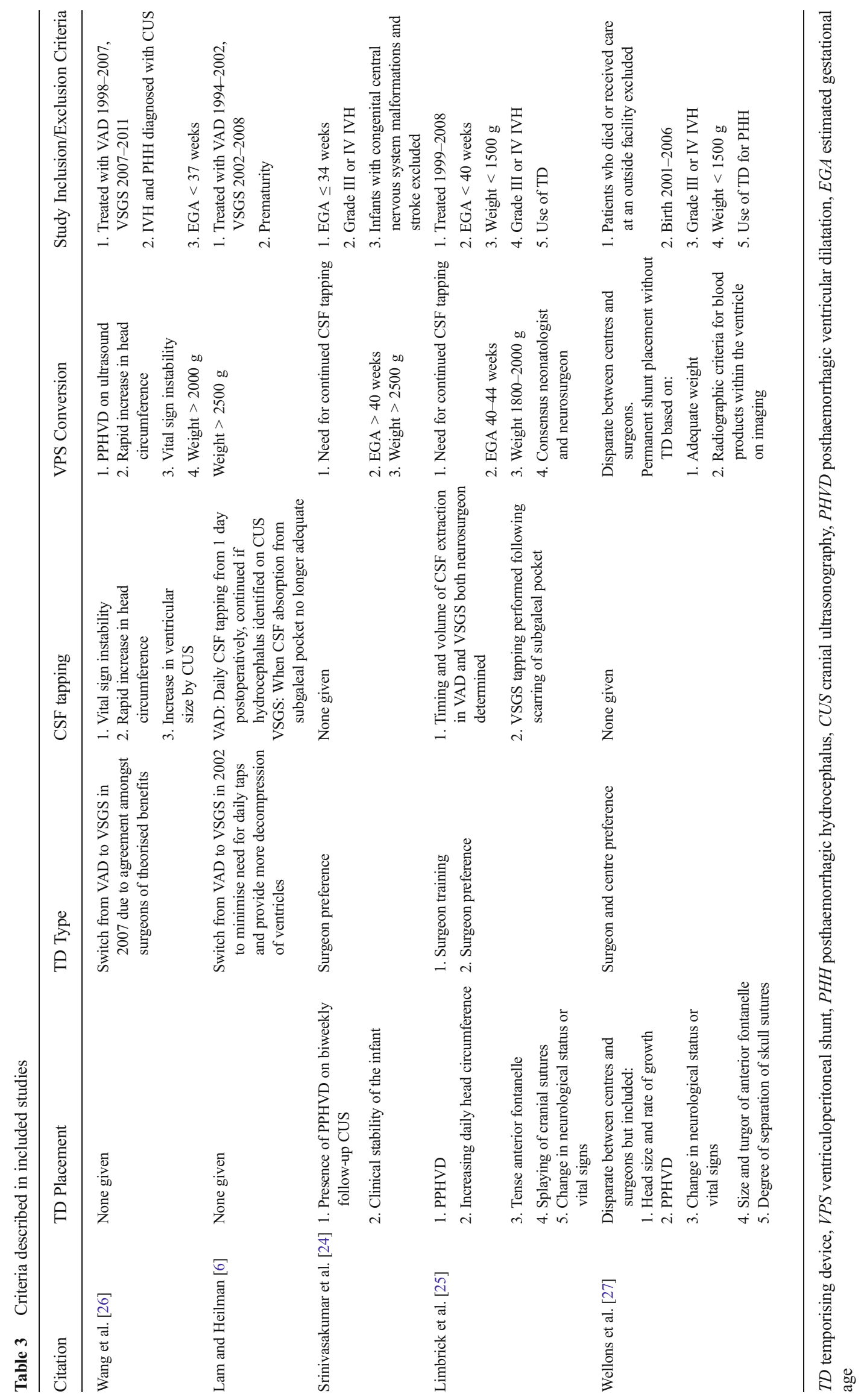


VSGS) is superior for the management of PHH in preterm infants. The systematic search revealed an absence of randomised controlled trials investigating this clinical equipoise. Five studies of poor quality (three class $3 \mathrm{~b}$ studies and two class 4 studies) were identified and reviewed, with an observational study meta-analysis performed. There is evidence to suggest fewer CSF tappings are necessary with a ventriculosubgaleal shunt. Meta-analysis showed no significant differences between VAD and VSGS in rates of temporising device infection, obstruction, the requirements for a ventriculoperitoneal shunt, infection of the subsequent shunt and overall mortality. Heterogeneity of included studies reaffirms the importance of standardised criteria for initial management and reporting outcomes, along with a more systematic approach to sustained follow-up to enable a better understanding of long-term neurodevelopment of these patients. There is not current sufficient data to suggest superiority of one TD over the other, although the reduced rate of CSF tapping in VSGS may make this option attractive in certain healthcare systems.

Acknowledgments The authors would like to thank Isla Kuhn at the Medical Library of the University of Cambridge for her support in the development of the search strategy and Dominic Thompson (Great Ormond Street Hospital) for the comments on the manuscript. The authors received no financial and/or material support for the research reported in this paper.

Open Access This article is distributed under the terms of the Creative Commons Attribution 4.0 International License (http:// creativecommons.org/licenses/by/4.0/), which permits unrestricted use, distribution, and reproduction in any medium, provided you give appropriate credit to the original author(s) and the source, provide a link to the Creative Commons license, and indicate if changes were made.

\section{References}

1. Tsitouras V, Sgouros S (2011) Infantile posthemorrhagic hydrocephalus. Childs Nerv Syst ChNS Off J Int Soc Pediatr Neurosurg 27:1595-1608. doi:10.1007/s00381-011-1521-y

2. Maunu J, Lehtonen L, Lapinleimu H, et al. (2011) Ventricular dilatation in relation to outcome at 2 years of age in very preterm infants: a prospective Finnish cohort study. Dev Med Child Neurol 53:48-54. doi:10.1111/j.1469-8749.2010.03785.x

3. Persson E-K, Hagberg G (1992) Uvebrant P (2005) Hydrocephalus prevalence and outcome in a population-based cohort of children born in 1989-1998. Acta Paediatr Oslo Nor 94:726-732. doi:10. 1080/08035250510027336

4. Robinson S (2012) Neonatal posthemorrhagic hydrocephalus from prematurity: pathophysiology and current treatment concepts. J Neurosurg Pediatr 9:242-258. doi:10.3171/2011.12.PEDS11136

5. van Haastert IC, Groenendaal F, Uiterwaal CSPM, et al. (2011) Decreasing incidence and severity of cerebral palsy in prematurely born children. J Pediatr 159:86-91.e1. doi:10.1016/j.jpeds.2010. 12.053
6. Taylor AG, Peter JC (2001) Advantages of delayed VP shunting in post-haemorrhagic hydrocephalus seen in low-birth-weight infants. Childs Nerv Syst ChNS Off J Int Soc Pediatr Neurosurg 17:328333

7. Kulkarni AV, Drake JM, Lamberti-Pasculli M (2001) Cerebrospinal fluid shunt infection: a prospective study of risk factors. J Neurosurg 94:195-201. doi:10.3171/jns.2001.94.2.0195

8. Lam HP, Heilman CB (2009) Ventricular access device versus ventriculosubgaleal shunt in post hemorrhagic hydrocephalus associated with prematurity. J Matern-Fetal 22:1097-1101. doi:10. 3109/14767050903029576

9. Tubbs RS, Banks JT, Soleau S, et al. (2005) Complications of ventriculosubgaleal shunts in infants and children. Childs Nerv Syst 21:48-51

10. Whitelaw A (2001) Repeated lumbar or ventricular punctures in newborns with intraventricular hemorrhage. Cochrane Database Syst, Rev

11. Mazzola CA, Choudhri AF, Auguste KI, et al. (2014) Pediatric hydrocephalus: systematic literature review and evidence-based guidelines. Part 2: management of posthemorrhagic hydrocephalus in premature infants. J Neurosurg Pediatr 14:8-23

12. McComb J, Ramos A, Platzker A, et al. (1983) Management of hydrocephalus secondary to intraventricular hemorrhage in the preterm infant with a subcutaneous ventricular catheter reservoir. Neurosurgery 13:295-300

13. Hu YC, Chowdhry SA, Robinson S (2011) Infantile posthemorrhagic hydrocephalus. In: Youmans Neurol. Surg., 6 edition. Saunders, pp 1989-1991

14. Fulmer BB, Grabb PA, Oakes WJ, Mapstone TB (2000) Neonatal ventriculosubgaleal shunts. Neurosurgery 47:80-83 discussion $83-$ 84

15. Koksal V, Oktem S (2010) Ventriculosubgaleal shunt procedure and its long-term outcomes in premature infants with post-hemorrhagic hydrocephalus. Childs Nerv Syst 26:1505-1515. doi:10.1007/ s00381-010-1118-x

16. Tubbs RS, Smyth MD, Wellons JC, et al. (2003) Life expectancy of ventriculosubgaleal shunt revisions. Pediatr Neurosurg 38:244246. doi: 69827

17. Willis BK, Kumar CR, Wylen EL, Nanda A (2005) Ventriculosubgaleal shunts for posthemorrhagic hydrocephalus in premature infants. Pediatr Neurosurg 41:178-185. doi:10.1159/ 000086558

18. Sklar F, Adegbite A, Shapiro K, Miller K (1992) Ventriculosubgaleal shunts: management of posthemorrhagic hydrocephalus in premature infants. Pediatr Neurosurg 18:263-265

19. Kadri H, Mawla AA, Kazah J (2006) The incidence, timing, and predisposing factors of germinal matrix and intraventricular hemorrhage (GMH/IVH) in preterm neonates. Childs Nerv Syst ChNS Off J Int Soc Pediatr Neurosurg 22:1086-1090. doi:10.1007/ s00381-006-0050-6

20. Howick J (2009) Oxford Centre for Evidence-based Medicinelevels of evidence. University of Oxford, Centre for EvidenceBased Medicine

21. von Elm E, Altman DG, Egger M, et al. (2014) The Strengthening the Reporting of Observational Studies in Epidemiology (STROBE) statement: guidelines for reporting observational studies. Int J Surg 12:1495-1499

22. Stroup DF, Berlin JA, Morton SC, et al. (2000) Meta-analysis of observational studies in epidemiology: a proposal for reporting. Meta-analysis Of Observational Studies in Epidemiology (MOOSE) group. JAMA 283:2008-2012

23. Cochran WG (1954) The combination of estimates from different experiments. Biometrics 10:101-129. doi:10.2307/3001666

24. Development Core Team R (2008) R: a language and environment for statistical computing. R Foundation for Statistical Computing. R Foundation for Statistical Computing, Vienna, Austria 
25. Viechtbauer W (2010) Conducting meta-analyses in R with the metafor package. J Stat Softw 36:1-48

26. Srinivasakumar P, Limbrick D, Munro R, et al. (2013) Posthemorrhagic ventricular dilatation-impact on early neurodevelopmental outcome. Am J Perinatol 30:207-214. doi: $10.1055 / \mathrm{s}-0032-1323581$

27. Limbrick DDJ, Mathur A, Johnston JM, et al. (2010) Neurosurgical treatment of progressive posthemorrhagic ventricular dilation in preterm infants: a 10-year single-institution study. J Neurosurg Pediatr 6:224-230. doi:10.3171/2010.5.PEDS1010

28. Wang JY, Amin AG, Jallo GI, Ahn ES (2014) Ventricular reservoir versus ventriculosubgaleal shunt for posthemorrhagic hydrocephalus in preterm infants: infection risks and ventriculoperitoneal shunt rate: clinical article. J Neurosurg Pediatr 14:447-454

29. Wellons JC, Shannon CN, Kulkarni AV, et al. (2009) A multicenter retrospective comparison of conversion from temporary to permanent cerebrospinal fluid diversion in very low birth weight infants with posthemorrhagic hydrocephalus. J Neurosurg 4:50-55. doi:10.3171/2009.2.PEDS08400

30. Brouwer A, Brouwer M, Groenendaal F, et al. (2012) European perspective on the diagnosis and treatment of posthaemorrhagic ventricular dilatation. Arch Dis Child - Fetal Neonatal Ed 97: F50-F55. doi:10.1136/adc.2010.207837

31. Spiegelman L, Asija R, Da Silva SL, et al. (2014) What is the risk of infecting a cerebrospinal fluid-diverting shunt with percutaneous tapping? J Neurosurg Pediatr 14:336-339. doi:10.3171/2014.7. PEDS13612

32. University of Utah Shunt Outcomes of Post-Hemorrhagic Hydrocephalus (SOPHH). In: ClinicalTrials.gov. https:// clinicaltrials.gov/ct2/show/NCT01480349?term=PostHemorrhagic + Hydrocephalus\&rank=1. Accessed 24 Apr 2015 